\title{
Suppression of three-dimensional flow instabilities in tube bundles
}

\author{
N. K.-R. Kevlahan* \\ Department of Mathematics \& Statistics, McMaster University, Hamilton, Canada \\ J. Wadsley \\ Department of Physics \& Astronomy, McMaster University, Hamilton, Canada
}

\begin{abstract}
We study the generation of three-dimensional vorticity in tightly packed tube bundles. In particular, our goal is to investigate which conditions (if any) enable the flow to remain two-dimensional for $\operatorname{Re}>180$. We calculated two- and three-dimensional flow through periodic rotated square tube bundles with tight packing, $P / D=1.5$, using a high resolution pseudo-spectral code with penalization. The tubes are cylinders whose response is modelled as a rigid harmonic oscillator forced by the flowinduced lift. We find that at $\operatorname{Re}=200$ tube motion completely suppresses the three-dimensional instability. At $\mathrm{Re}=1000$ tube motion does not suppress the three-dimensional instability, although the flow does have increased spanwise correlation and the Strouhal number for the two- and three-dimensional flows is approximately the same. The tight packing alone does not suppress the three-dimensional instability. Three-dimensional vorticity drastically reduces fluid forces acting on the tube compared with an equivalent two-dimensional flow.
\end{abstract}

\section{Introduction}

The wake of an isolated cylinder first becomes three-dimensional at $\operatorname{Re} \approx 180$ via the formation of regular streamwise vortices with a spacing of about three cylinder diameters [the mode A instability identified by Williamson (1989)]. At $\mathrm{Re} \approx 230$ a second vortex mode appears [the mode B instability identified by Williamson (1989)], characterized by irregular streamwise vortices with

* Corresponding author.

Email address: kevlahan@mcmaster.ca (N. K.-R. Kevlahan). 
a spacing of one cylinder diameter (Williamson, 1989). As Reynolds number increases further, the wake becomes increasingly complicated (possibly via period-doubling) until it is completely turbulent. In contrast, the transition to three-dimensionality in tightly coupled tube bundles is still not well understood. Indeed, in some experiments it appears that the flow and cylinder response remain roughly two-dimensional for Reynolds numbers well beyond 180 (Weaver, 2001). For example, Price et al. (1995) find that Strouhal frequency and rms drag do not change greatly with Reynolds number for Re $>150$. Blevins (1985) demonstrated that acoustic forcing of an isolated cylinder at its Strouhal frequency is able to produce nearly perfect spanwise correlation of pressure for $20000 \leq R e \leq 40000$. He conjectured that similar effects might be observed in tube bundles. Blevins' investigations confirmed earlier work by Toebes (1969) who showed that a cylinder vibration amplitude of $A / D \geq 0.125$ was required to achieve a high degree of spanwise correlation. In the case of tube bundles, it has also been suggested that it is the tight spacing of the bundle that suppresses three-dimensional instability.

In this paper we attempt to understand which conditions (if any) enable the flow to remain two-dimensional for $\operatorname{Re}>180$. It is also possible that some aspects of the flow remain two-dimensional (e.g. spanwise correlation of vortex shedding), while others become fully three-dimensional (e.g. development of streamwise vorticity). To investigate these questions we calculate two- and three-dimensional flow through periodic rotated square tube bundles with spacing $P / D=1.5$. We consider two types of bundles: fixed cylinder and moving cylinder bundles. The natural frequency of the moving cylinders is tuned to match the Strouhal frequency in order to maximize the moving cylinder effect. All cylinders move in phase, which corresponds to the acoustic resonance conditions investigated by Blevins (1985).

The mathematical formulation of the problem is described in Section 2 and the penalized pseudo-spectral method used to solve the equations is outlined in Section 3. The simulations are described in Section 4 and results are presented in Section 5. The main conclusions are summarized in Section 6.

\section{Problem formulation}

Let us consider a viscous incompressible fluid governed by the Navier-Stokes equations

$$
\begin{aligned}
\frac{\partial \mathbf{u}}{\partial t}+\left(\mathbf{u}+\mathbf{U}_{\infty}\right) \cdot \nabla \mathbf{u}+\nabla P & =\nu \Delta \mathbf{u}, \\
\nabla \cdot \mathbf{u} & =0,
\end{aligned}
$$


where $\mathbf{u}$ is the velocity, $P$ is the pressure and $\mathbf{U}_{\infty}$ is an imposed upstream flow velocity. We focus here on the case where the fluid occupies the complement in the plane $R^{3}$ of a periodic lattice of cylinders $O_{i}$ (with diameter $D)$ oriented with their axes in the $x_{3}$ direction. The external boundary conditions associated with this problem are therefore that $\mathbf{u}$ is $Q$-periodic on ] $0, L_{1}[\times] 0, L_{2}[\times] 0, L_{3}[$, with no-slip boundary conditions on the surface of the (moving) obstacle,

$$
\mathbf{u}+\mathbf{U}_{\infty}=\mathbf{U}_{o} \text { on } \partial O_{i}, \forall i
$$

where $\mathbf{U}_{o}$ is the velocity of the obstacle. Note that all quantities (e.g. Strouhal number, Reynolds number and time) will be nondimensionalized using the upstream flow velocity $\mathbf{U}_{\infty}$, the cylinder diameter $D$ and the kinematic viscosity $\nu$.

To model the no-slip boundary conditions without explicitly imposing Eqs. (3) we follow Angot et al. (1999) by replacing Eqs. (1-3) by the following set of $L^{2}$-penalized equations:

$$
\begin{aligned}
\frac{\partial \mathbf{u}_{\eta}}{\partial t}+\left(\mathbf{u}_{\eta}+\mathbf{U}_{\infty}\right) \cdot \nabla \mathbf{u}_{\eta}+\nabla P_{\eta} & =\nu \Delta \mathbf{u}_{\eta}-\frac{1}{\eta} \chi(\mathbf{x}, t)\left(\mathbf{u}_{\eta}+\mathbf{U}_{\infty}-\mathbf{U}_{o}\right), \\
\nabla \cdot \mathbf{u}_{\eta} & =0
\end{aligned}
$$

where $\mathbf{U}_{o}$ is the obstacle's velocity. Note that Eqs. (4-5) are valid in the entire domain $\Omega$ : the last term on the right hand side of (4) is a volume penalization of the flow inside the obstacle. Here $0<\eta \ll 1$ is a penalization coefficient and $\chi$ denotes the characteristic function (or mask)

$$
\chi(\mathbf{x}, t)= \begin{cases}1 & \text { if } \mathbf{x} \in O_{i} \\ 0 & \text { otherwise }\end{cases}
$$

This approximation is called Brinkman penalization, and models flow through a porous medium with fixed porosity and (very small) permeability $\eta$. Angot (1999) proved that the solution of the penalized Eqs. (4-5) converge to that of the Navier-Stokes Eqs. (1-2) with the correct boundary conditions (3) as $\eta \rightarrow$ 0 . More precisely, the upper bound on the global error of the $L^{2}$-penalization was shown to be (Angot, 1999)

$$
\left\|\mathbf{u}-\mathbf{u}_{\eta}\right\|_{H^{1}(\Omega)}=O\left(\eta^{1 / 4}\right) .
$$

In the specific case of impulsively started flow over a plane Kevlahan and Ghidaglia (2001) showed analytically that the error in approximating (3) is 
actually lower: $O\left(\eta^{1 / 2}\right)$. It seems reasonable that this is the sharp estimate in the general case as well. Note that the velocity is continuous and differentiable, due to the instantaneous smoothing action of the Laplacian operator term (i.e. viscous diffusion).

This volume penalization has been implemented in a finite difference code (Khadra et al., 2000) for two-dimensional flow around an isolated cylinder, and was found to give good results. It is important to note that $\eta$ is an arbitrary parameter, independent of the spatial or temporal discretization, and thus the boundary conditions can be enforced to any desired accuracy by choosing $\eta$ appropriately. This property distinguishes the Brinkman method from other penalization schemes and allows the error to be controlled precisely.

Another advantage of the Brinkman penalization is that the force $\mathbf{F}_{i}$ acting on an obstacle $O_{i}$ can be found by simply integrating the penalization term over the volume of the obstacle:

$$
\mathbf{F}_{i}=\frac{1}{\eta} \int_{O_{i}}\left(\mathbf{u}_{\eta}+\mathbf{U}_{\infty}-\mathbf{U}_{o}\right) \mathrm{d} \mathbf{x} .
$$

Thus, the calculation of lift and drag on an obstacle can be made simply, accurately and at low cost. This is helpful when calculating fluid-structure interaction, where the force must be updated at each time step. Kevlahan and Ghidaglia (2001) showed analytically that the error in calculating the force over a flat plate using (8) is only $O(\eta)$. We have found numerically that $\eta=10^{-4}$ gives drag curves correct to within $1 \%$.

The cylinders move as forced simple harmonic oscillators according to the equation

$$
\left(m+m_{A}\right) \frac{d^{2} \mathbf{x}_{o}}{d t^{2}}+b \frac{d \mathbf{x}_{o}}{d t}+k \mathbf{x}_{o}=\mathbf{F}_{w}(t),
$$

where $\mathbf{x}_{o}(t)$ is the cylinder position, $m$ is the cylinder mass, $m_{A}$ is the added mass, $b$ is the damping, $k$ is the spring constant. The fluid forcing $\mathbf{F}_{w}(t)$ (due to wake vorticity) is calculated as a volume integral using (8). Thus the penalized Navier-Stokes Eqs. (4-5) are fully coupled to the cylinder motion equation (9) via the fluid force Eq. (8).

In this paper take the cylinder diameter $D=1$, and consider square cylinder arrays where the ratio of pitch (cylinder spacing) to diameter $P / D=1.5$ (tightly packed). Figure 1 shows the tube bundle geometry. This configuration models flow in the interior of a very large periodic array. The periodic domain contains only one cylinder, and thus all (image) cylinders move in phase. This corresponds to the case of acoustic resonance, caused by a standing acoustic 




Fig. 1. Tube bundle geometry. Note that there is one tube per periodic domain (indicated by dashed lines), and the mean flow is at $45^{\circ}$ to the array axis (i.e. this is a staggered or rotated square array). The tubes are free to oscillate in the transverse direction only.

wave between the duct walls. Although modelling all cylinders as moving in phase is unrealistic for some flows, it should maximize any suppression of three-dimensional instabilities via spanwise correlation of the vorticity. The cylinders move freely in the transverse direction due to the fluid-induced lift force.

\section{Numerical method}

The penalized Navier-Stokes equations are solved using a Fourier-transformbased pseudo-spectral method in space [e.g. Vincent and Meneguzzi (1991)] and a Krylov method in time (Edwards et al., 1994). The pseudo-spectral method is computationally efficient and highly accurate for spatial derivatives, while the Krylov method is a stiffly stable explicit method with an adaptive stepsize to maintain error to a specified tolerance.

In the pseudo-spectral method derivatives are calculated in Fourier space with exponential accuracy, while nonlinear terms (i.e. the advection and penalization terms) are calculated in physical space. This approach has zero numerical dissipation, which is important for accurate simulation of moderate to high Reynolds number calculations. The incompressibility condition is enforced by projecting the velocity in Fourier space onto the plane normal to the wave vector $\mathbf{k}$. A constant equivalent upstream flow $\mathbf{U}_{\infty}$ is imposed by setting the time evolution of the zero wavenumber mode to zero. Note that the pitch velocity $\mathbf{U}_{p}$ (i.e. the average velocity between the tubes) is greater than $\mathbf{U}_{\infty}$ : $\mathbf{U}_{p}=\mathbf{U}_{\infty}\left(V_{\Omega} / V_{F}\right)$, where $V_{\Omega} / V_{F}$ is the ratio of total volume to fluid volume. The difference between $\mathbf{U}_{\infty}$ and $\mathbf{U}_{p}$ should be remembered when comparing the present results to those for isolated cylinders. All quantities are nondimen- 
sionalized with respect to $\mathbf{U}_{\infty}$. Note that enforcing a constant upstream flow is equivalent to adding energy to the system to compensate the energy lost to dissipation.

Although the mask $\chi(\mathbf{x}, t)$ used to define the position of the tubes is discontinuous, the velocity remains continuous and differentiable. This ensures that Gibbs oscillations are small, and we have found they do not perturb the calculation, even in the vorticity formulation (Kevlahan and Ghidaglia, 2001). One of the main advantages of the Brinkman penalization is that it allows us to use a Cartesian (rectangular) computational grid, which is computationally efficient. By performing grid convergence studies, we have found that a grid spacing of $h=\delta / 6$ in the wall normal direction (where $\delta=\operatorname{Re}^{-1 / 2}$ is the boundary layer thickness) is sufficient to give fully converged results.

The three-dimensional calculations are large $\left(288^{2} \times 96\right.$ grid points $)$ and time consuming (especially at $\mathrm{Re}=1000$ ), and so we have developed a parallel version of the code. The Fourier transforms are performed using FFTw (Frigo and Johnson, 1998), which parallelizes the calculation by decomposing the domain into spanwise slabs (one per processor). The Krylov time scheme is also parallelized using MPI. The resulting code scales surprisingly well for such a tightly coupled calculation: for example, each time step is 2.5 times faster on 48 processors than on 16. All parallel calculations were carried on McMaster University's SHARCNET cluster IDRA, which has 128 processors linked by a Quadrics network.

One drawback of the penalization approach is that the small parameter $\eta$ makes the equations stiff. Because of this stiffness, stepsizes are bounded by the penalization parameter, i.e $\Delta t<\eta$, unless we employ a stiffly stable method in time. To obtain an accurate force calculation we must also use a highorder method that automatically adjusts the time step to maintain the desired tolerance. We have found that the Krylov time-stepping method developed by Edwards et al. (1994) works very well for the present problem. This approach is based on the Krylov method for solving a linear system, and its accuracy (in the linear case) is $O(K)$, where $K$ is the dimension of the Krylov subspace used to approximate the matrix representing the right hand side of the equation. We have found that $K=10$ is optimal for computational efficiency, and gives sufficient accuracy. The step size is set to ensure that the $L^{2}$ error in the approximation of $\partial \mathbf{u} / \partial t$ is smaller than the desired tolerance. This is done by comparing the exact value for $\partial \mathbf{u} / \partial t$ (given by the right hand side of the equation) to the approximation to $\partial \mathbf{u} / \partial t$ given by differentiating the Krylov approximation to $\mathbf{u}(t)$. We have found that the Krylov approach gives good results, especially when the cylinder is moving. In our calculations we use $K=10$, and set the $L^{2}$ error tolerance to $10^{-3}$. With these parameters we achieve a CFL value of 2 to 6 . 




Fig. 2. Drag for impulsively started flow past a cylinder at $\operatorname{Re}=550$.

To eliminate noise in the force calculation when the cylinder is moving which is caused when grid points move in or out of the mask, we slightly smooth the edge of the mask. The mask is smoothed over a distance of only $h / 6$ (i.e. $1 / 6$ of a grid point). This smoothing completely eliminates the noise, and changes the force by less than $1 \%$. One can view this slight smoothing as rounding the step-like edge of the cylinder due to the Cartesian grid approximation of its boundary. Figure 2 shows the drag for impulsively started flow around a two-dimensional cylinder at $\mathrm{Re}=550$ calculated with the present method (on a large $L_{1}=L_{2}=20$ domain so $L_{1} \gg \mathbf{U}_{\infty} T$ ) compared with results from a vortex method (Koumoutsakos and Leonard, 1995), and the short time asymptotic solution (Bar-Lev and Yang, 1975). The good agreement demonstrates that our penalized pseudo-spectral method gives reliable force results.

\section{Simulations}

In each of the following cases we do four simulations: two-dimensional with fixed and moving cylinders, and three-dimensional with fixed and moving cylinders. This allows us to directly compare the two- and three-dimensional flows in order to determine the degree of suppression of three-dimensional instability. The Reynolds number is defined as $\operatorname{Re}=\left|\mathbf{U}_{\infty}\right| D / \nu$ (where the upstream velocity $\left|\mathbf{U}_{\infty}\right|=1$, and the cylinder diameter $D=1$ ), and the flow is at $45^{\circ}$ to the axis of the square cylinder array (this is referred to as a rotated square array). The computational domain contains one cylinder and has dimensions $L_{1} \times L_{2}=1.5 \times 1.5$ in the plane perpendicular to the 
Table 1

\begin{tabular}{rclllll}
\hline Re & Dimension & Resolution & $L_{3}$ & $m_{*}$ & $b$ & $f$ \\
\hline 200 & 2 -D & $128^{2}$ & 6.0 & 5 & 0 & 0.98 \\
200 & 3 -D & $128^{2} \times 64$ & 6.0 & 5 & 0 & 0.98 \\
1000 & $2-\mathrm{D}$ & $288^{2}$ & 1.5 & 5 & 0 & 1.00 \\
1000 & $3-\mathrm{D}$ & $288^{2} \times 96$ & 1.5 & 5 & 0 & 1.00 \\
\hline
\end{tabular}

Parameters for the numerical simulations. Fixed and moving cylinder runs are done for each of the above cases.

cylinder axis (see Figure 1). In each case the spring frequency $f$ is chosen to match the Strouhal frequency of the fixed two-dimensional cylinder, the cylinder is undamped $(b=0)$, and the nondimensional tube mass per unit length is $m_{*}=m /\left(\rho D^{2} / 2\right)=5$. We set the total nondimensional mass to be $m_{*}+\pi / 2$ (where $\pi / 2=m_{A}^{*}$ is the nondimensional added mass), and thus the tube is forced by the wake force only, as in Shiels et al. (2001). The parameters for each simulation are summarized in Table 1 . Note that we consider two Reynolds numbers: $R e=200$ (just above the mode A three-dimensional transition), and $R e=1000$ (when the flow should be fully three-dimensional). This allows us to compare the effect of tube motion on weakly and strongly three-dimensional flows.

The computational grid is $128^{2} \times 64$ for the $\mathrm{Re}=200$ simulations, and the length of the domain in the spanwise direction is 6 (sufficient to capture the streamwise vorticity, which has a wavelength of about $3-D)$. Although the spanwise direction is not as finely resolved as the other directions $(h=3 / 4 \delta$, compared with $h=\delta / 6$ for the other directions), we have checked a posteriori that three-dimensional vorticity is sufficiently well resolved. This is actually slightly more than the spanwise resolution used in the finite volume calculations of Persillon and Braza (1998). The normalized spring constant is set to $k_{*}=k /\left(\rho U_{\infty}^{2} / 2\right)=451.1$ in order to match the Strouhal number $S_{t}=1.32$ of the fixed two-dimensional cylinder.

At $\operatorname{Re}=1000$ we kept the same streamwise resolution as for $\operatorname{Re}=200$ (i.e. $h=\delta / 6)$, but found that we needed to increase the spanwise resolution to $h=\delta / 2$. The computational grid is thus $288^{2} \times 96$, and the length of the domain in the spanwise direction is 1.5 (i.e. the domain is cubic). As we will see, the shorter spanwise domain is sufficient due to the smaller size of the streamwise vortices at this Reynolds number. The normalized spring constant is set to $k_{*}=130$ in order to match the peak Strouhal number St $=1.00$ of the fixed two-dimensional cylinder. 

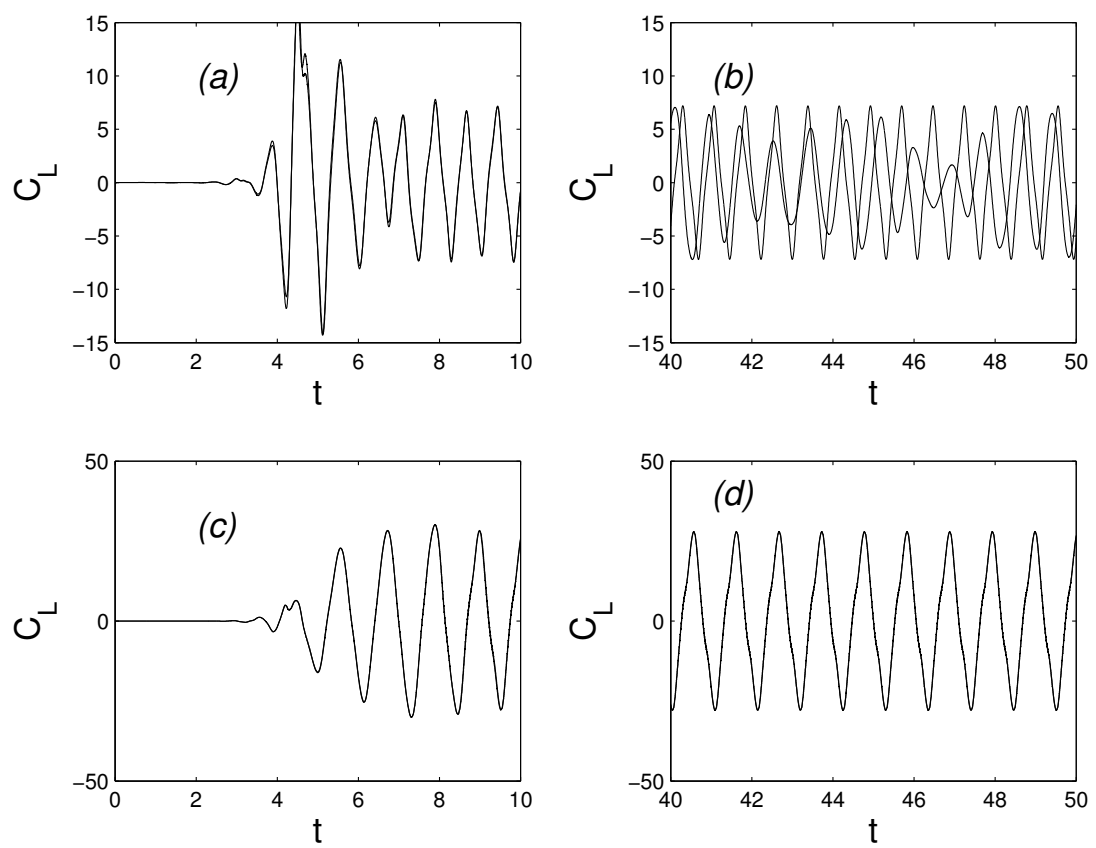

Fig. 3. Lift curves for $\mathrm{Re}=200$ case. (a) Fixed cylinder, short times: 2-D and 3-D results match. (b) Fixed cylinder, long times: 2-D has fixed amplitude, while 3-D has modulated amplitude. (c) Moving cylinder, short times: 2-D and 3-D results match. (d) Moving cylinder, long times: 2-D and 3-D results match.

\section{Results}

\subsection{Reynolds number 200}

Figure 3 shows the lift curves at short and long times for the fixed and moving cylinders at $R e=200$. In both cases the lift curves agree at short times (before the three-dimensional instability has developed). However, at longer times the three-dimensional fixed cylinder's lift amplitude is modulated in a nonstationary way, whereas the the moving cylinder's lift still closely matches that of the moving two-dimensional cylinder. Table 2 lists the peak lift frequencies for each of the cases. The frequencies of the two- and three-dimensional moving cylinder cases match, while those of the fixed cases differ by about 0.14 . Note that the Strouhal number of the three-dimensional fixed tube is 1.18. This value is much higher than the experimental value of 0.18 for an isolated tube (Williamson, 1989), but is consistent with the value of $1.25 \pm 0.1$ found for rotated square tube bundles with $P / D=1.5$ by Price et al. (1995).

Figure 4 shows the vorticity field at $t=50$ (the point of maximum lift during the vortex shedding cycle). It is clear from Figure 4(c) that the moving cylinder completely suppresses the production of streamwise vorticity, while Figure 4(a) shows the development of streamwise vortices at a spacing of about 

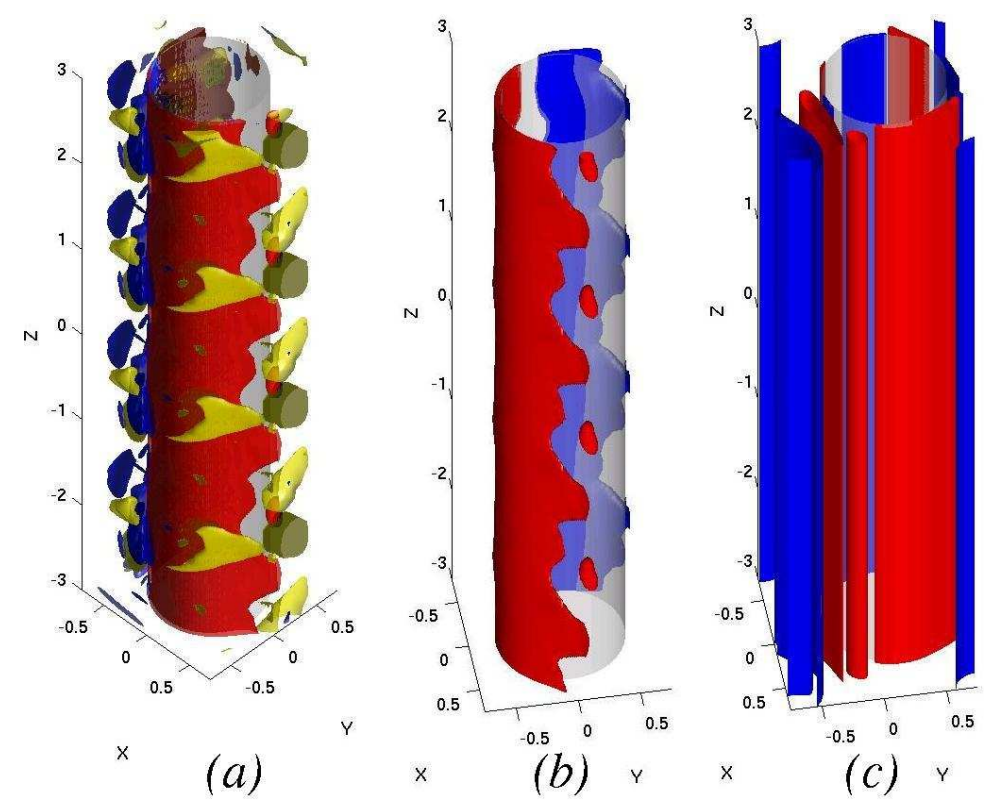

Fig. 4. Isosurfaces of three-dimensional vorticity at $t=50$ for $\operatorname{Re}=200$. The spanwise vorticity isosurfaces are at 0.25 of maximum vorticity magnitude, and the transverse and streamwise vorticity isosurfaces are at $0.25 / 4$ of maximum vorticity. (a) Fixed cylinder, three components. Four streamwise vortices are evident, with a spacing of about $1.5 \mathrm{D}$. (b) Fixed cylinder, spanwise vorticity only. (c) Moving cylinder, spanwise vorticity only.

$1.5 D$, twice as close as the spacing of $3 D$ seen in simulations of flow past an isolated cylinder (Persillon and Braza, 1998). In fact, the spacing observed here is much closer to the mode B spacing of about $1 D$. Finally, Figure 4(b) shows that, although significant streamwise vorticity has been generated, the spanwise vorticity is still largely two-dimensional except for some perturbation at the trailing edge of the vortex sheets due to interaction with the streamwise vortices.

These results show that at $\mathrm{Re}=200$ the in-phase 'acoustic resonance' movement of the cylinders completely suppresses the development of any threedimensionality in the flow. Since the amplitude of cylinder oscillation is $A / D=$ $0.23>0.125$, these results are consistent with the observations of Toebes (1969) that sufficiently large amplitude cylinder vibration amplitude should produce a high degree of spanwise correlation. We also find that the tight packing alone does not suppress three-dimensional instabilities.

\subsection{Reynolds number 1000}

The drag and lift curves for the $\mathrm{Re}=1000$ simulations shown in Figure 5 demonstrate that the three-dimensional forces are decorrelated from the twodimensional forces, and are of much lower amplitude for both the fixed and 
Table 2

\begin{tabular}{lll}
\hline Case & $\mathrm{Re}=200$ & $\mathrm{Re}=1000$ \\
\hline 2-D, fixed & 1.32 & 1.08 \\
3-D, fixed & 1.18 & 0.92 \\
2-D, moving & 0.95 & 0.75 \\
3-D, moving & 0.95 & 0.80 \\
\hline
\end{tabular}

Strouhal numbers for each simulation. The result for the $R e=1000$ moving cylinder case is the average of the peaks at 0.92 and 0.67 .

moving cylinder cases. The small amplitude of the force fluctuations in the three-dimensional simulation is somewhat surprising. It is probably due to the streamwise vortices decorrelating the spanwise vorticity, and hence reducing the fluctuating force caused by vortex shedding (see Figure 6). The tight packing of the cylinders may enhance the decorrelating effect of streamwise vorticity. However, Figures 5(a,d) indicate that cylinder movement does still increase the spanwise correlation of spanwise vorticity compared with the fixed cylinder case. The very large force amplitudes of the two-dimensional simulation are clearly unphysical, as is seen from the fact that the two-dimensional drag is often negative (see Figures $5(\mathrm{a}, \mathrm{c})$ ).

The lack of suppression of the three-dimensional instability is consistent with the fact that, as shown in Figure 7, the cylinder vibration amplitude is only $A / D \approx 0.05$. This is less than the minimum threshold of 0.125 identified by Toebes (1969).

Somewhat surprisingly, as shown in Table 2 and Figure 8, the peak Strouhal frequency of the moving two-dimensional cylinder is approximately equal to the average of the two peak Strouhal frequencies of the moving three-dimensional cylinder. In contrast, the peak Strouhal frequencies of the fixed twoand three-dimensional cylinders differ by 0.16 . Recall that at $\operatorname{Re}=200$ the Strouhal numbers of two- and three-dimensional cylinders differ by a similar amount. These results show that, although three-dimensional vorticity is not suppressed by cylinder motion at $\mathrm{Re}=1000$, the average Strouhal numbers of the two-dimensional and three-dimensional flows are approximately the same. This suggests that cylinder motion decorrelates non-spanwise vorticity, reducing its effect on Strouhal number. Note also that the largest peak Strouhal frequency is the same for the moving and fixed three-dimensional cylinders. This shows that cylinder motion adds an additional shedding mode, but does not perturb the fixed cylinder shedding mode. Figure $6(\mathrm{a}, \mathrm{d})$ indicates that the spanwise vorticity is more strongly correlated in the moving cylinder case than in the fixed cylinder case. These results indicate that tuning the cylinder response to the Strouhal frequency of the fixed cylinder does not produce sufficiently large cylinder vibrations to suppress the three-dimensional instability. On the other hand, cylinder motion does reduce the effect of three-dimensional 

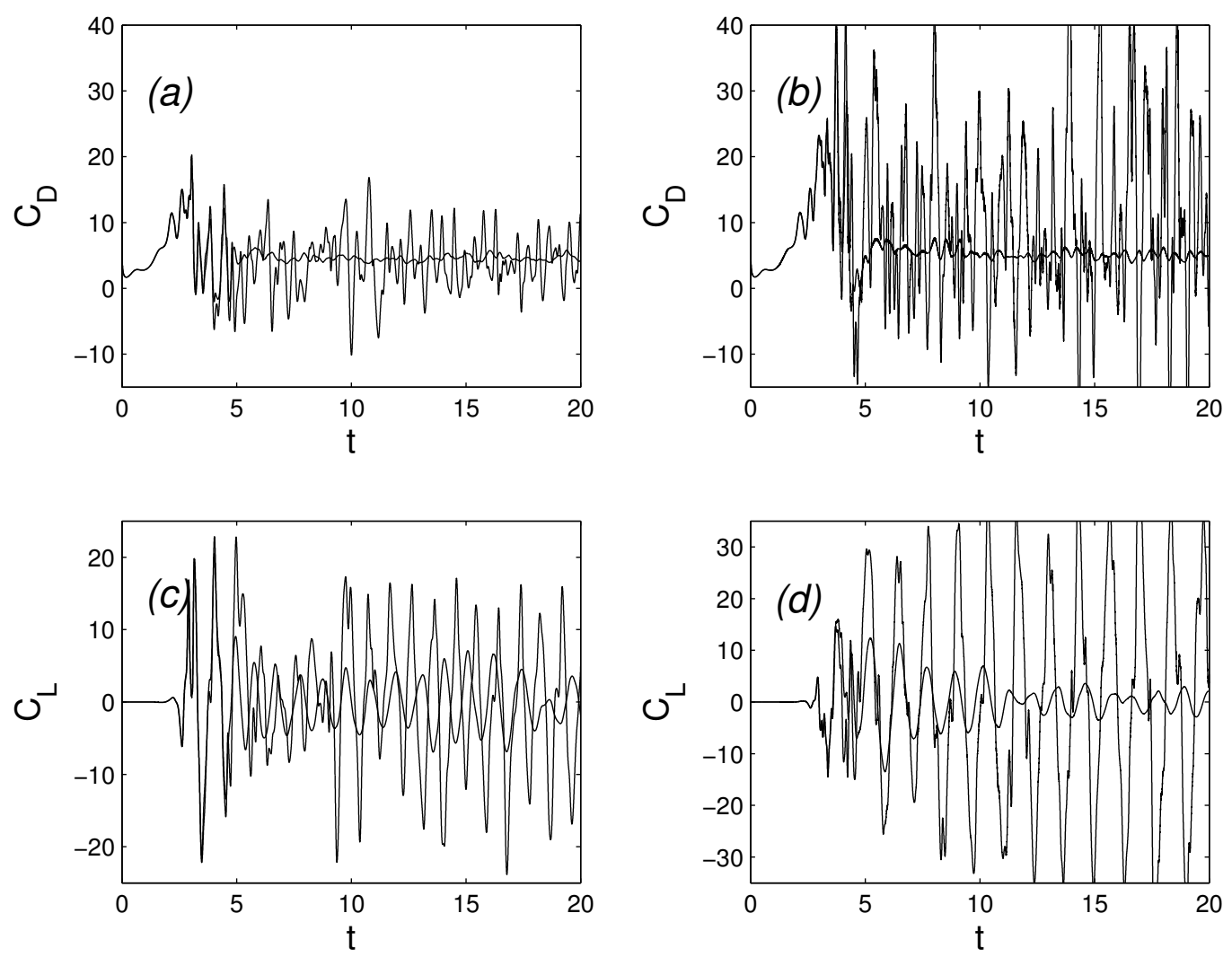

Fig. 5. Drag and lift at $R e=1000$ case. In each figure the three-dimensional curve is the one with the lower amplitude. (a) Drag, fixed cylinder. (b) Drag, moving cylinder. (c) Lift, fixed cylinder. (d) Lift, moving cylinder.

vorticity on the average Strouhal frequency.

\section{Conclusions}

We have used high resolution penalized pseudo-spectral simulations to investigate how and when in-phase cylinder vibration of tube bundles can suppress three-dimensionality. The in-phase cylinder vibration models the case of acoustic resonance, which was observed by Blevins (1985) to induce nearly perfect spanwise correlation of pressure for an isolated cylinder. We performed two- and three-dimensional simulations of fixed and free transverse vibrations of tube bundles at the relatively low Reynolds number $\operatorname{Re}=200$ (when only the mode A instability is present), and at the moderate Reynolds number $R e=1000$ (when the flow around the fixed cylinder is highly threedimensional and the three-dimensional instability is more complicated and much stronger).

We found that at $\operatorname{Re}=200$ the cylinder vibration is large enough $(A / D=$ 

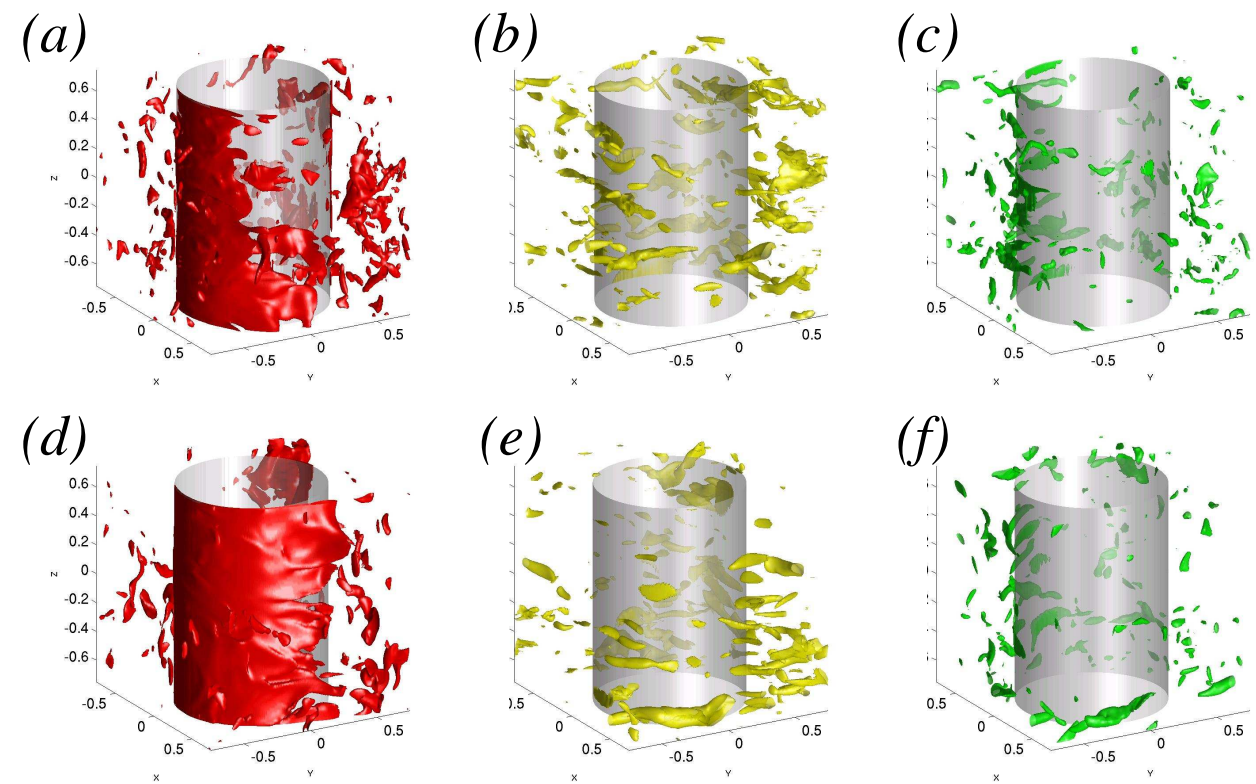

Fig. 6. Isosurfaces of three-dimensional vorticity magnitude at $t=15$ for $\operatorname{Re}=1000$. All vorticity isosurfaces are at 0.15 of maximum vorticity magnitude. (a) Fixed cylinder, spanwise vorticity. (b) Fixed cylinder, streamwise vorticity. (c) Moving cylinder, transverse vorticity. (d) Moving cylinder, spanwise vorticity. (e) Moving cylinder, streamwise vorticity. (f) Moving cylinder, transverse vorticity.
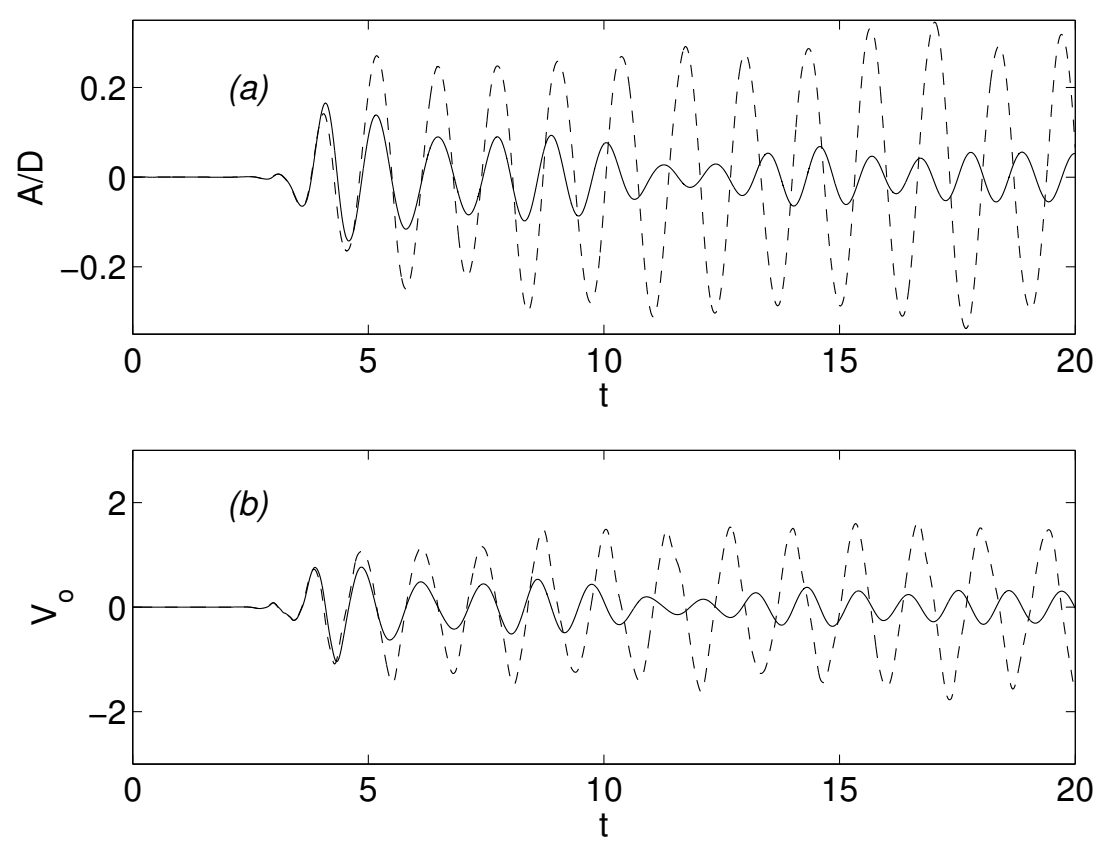

Fig. 7. Cylinder motion at $\operatorname{Re}=1000: 3-\mathrm{D},-; 2-\mathrm{D},-\cdots$ - . (a) Amplitude. (b) Velocity. 



Fig. 8. Lift spectra at $\operatorname{Re}=1000$ : (a) two-dimensional; (b) three-dimensional.

$0.23>0.125)$ to completely suppress the three-dimensional instability. The Strouhal frequency of the moving three-dimensional cylinder is therefore exactly the same as that of the moving two-dimensional cylinder, and no streamwise or transverse vorticity is produced. Note that the tight packing $P / D=1.5$ of the tube bundle is not sufficient by itself to suppress the three-dimensional instability.

In contrast, at $\operatorname{Re}=1000$ the cylinder vibration is insufficient $(A / D \approx 0.05<$ $0.125)$ to suppress the three-dimensional instabilities, although the spanwise vorticity is slightly more correlated. This may be due to the fact that significant transverse and streamwise vorticity (which is uncorrelated in the spanwise direction) is generated before the cylinder vibration is large enough to suppress the three-dimensional instability. This result does not agree with Blevins (1985)'s observation that acoustic forcing at the Strouhal frequency should correlate the flow in the spanwise directions, even at $20000 \leq R e \leq 40000$. In this case the tight packing of the tube bundle may actually reduce the cylinder vibration amplitude compared to the single cylinder case considered by Blevins. Despite the presence of three-dimensional vorticity, our results show that the average Strouhal frequencies of the two- and three-dimensional moving cylinder flows are similar. This is not true for the fixed cylinder case, and suggests that cylinder motion decorrelates non-spanwise vorticity so much that its effect on Strouhal frequency is negligible. In this sense, the moving cylinder flow at $\mathrm{Re}=1000$ may be described as partly two-dimensional. This effect is likely to persist for larger Reynolds numbers.

By comparing two- and three-dimensional simulations, we showed that threedimensional vorticity drastically reduces the amplitude of fluid forces on the cylinder (by as much as 10 times at $R e=1000$ ). This reduction in fluid forces produces a corresponding reduction in cylinder vibration amplitude. It is clear that the large forces of the two-dimensional flow at $\mathrm{Re}=1000$ are unphysical; indeed the drag is often negative in the two-dimensional case.

In summary, we have used two- and three-dimensional numerical simulations 
to show that resonant cylinder motion at $R e=200$ completely suppresses the generation of three-dimensional vorticity. At $R e=1000$ cylinder motion eliminates the effect of non-spanwise vorticity on the Strouhal number, although the generation of three-dimensional vorticity is not suppressed. Tight packing does not suppress the three-dimensional instability, and may actually reduce cylinder vibration amplitude.

\section{Acknowledgements}

The authors gratefully acknowledge the use of SHARCNET's parallel computers and an undergraduate SHARCNET fellowship. NKRK's research is supported by NSERC. We would like to thank J. Simon and N. Tonnet for help developing the parallel version of the code, and L. Tuckerman for suggesting the Krylov time integration method.

\section{References}

Angot, P., 1999. Analysis of singular perturbations on the brinkman problem for fictitious domain models of viscous flows. Mathematical Methods in the Applied Science 22, 1395-1412.

Angot, P., Bruneau, C.-H., Fabrie, P., 1999. A penalization method to take into account obstacles in viscous flows. Numerische Mathematik 81, 497520 .

Bar-Lev, M., Yang, H., 1975. Initial flow field over an impulsively started circular cylinder. Journal of Fluid Mechanics 72, 625-647.

Blevins, R., 1985. The effect of sound on vortex shedding. Journal of Fluid Mechanics 161, 217-237.

Edwards, W. S., Tuckerman, L. S., Friesner, R. A., Sorensen, D. C., 1994. Krylov methods for the incompressible Navier-Stokes equations. Journal of Computational Physics 110, 82-102.

Frigo, M., Johnson, S. G., 1998. FFTW: an adaptive software architecture for the FFT. In: ICASSP conference proceedings. Vol. 3. pp. 1381-1384.

Kevlahan, N., Ghidaglia, J.-M., 2001. Computation of turbulent flow past an array of cylinders using a spectral method with Brinkman penalization. European Journal of Mechanics/B 20, 333-350.

Khadra, K., Angot, P., Parneix, S., Caltagirone, J. P., 2000. Fictitious domain approach for numerical modelling of Navier-Stokes equations. International Journal of Numerical Methods in Fluids 34, 651-684.

Koumoutsakos, P., Leonard, A., 1995. High-resolution simulations of the flow around an impulsively started cylinder using vortex methods. Journal of Fluid Mechanics 296, 1-38. 
Persillon, H., Braza, M., 1998. Physical analysis of the transition to turbulence in the wake of a circular cylinder by three-dimensional Navier-Stokes simulation. Journal of Fluid Mechanics 365, 23-88.

Price, S. J., Païdoussis, M. P., Mark, B., 1995. Flow visualization of the interstitial cross-flow through parallel trangular and rotated square arrays of cylinders. Journal of Sound and Vibration 181, 85-98.

Shiels, D., Leonard, A., Roshko, A., 2001. Flow-induced vibration of a circular cylinder at limiting structural parameters. Journal of Fluids and Structures $15,3-21$.

Toebes, G., 1969. The unsteady flow and wake near an oscillating cylinder. Transactions of the ASME D: Journal of Basic Engineering 91, 493-498.

Vincent, A., Meneguzzi, M., 1991. The spatial structure and statistical properties of homogeneous turbulence. Journal of Fluid Mechanics 225, 1-20.

Weaver, D. S., 2001. Private communication.

Williamson, C. H. K., 1989. Oblique and parallel modes of vortex shedding in the wake of a circular cylinder at low reynolds numbers. Journal of Fluid Mechanics 206, 579-627. 